\title{
Dark- and bright-rogue-wave solutions for media with long-wave-short-wave resonance
}

\author{
Shihua Chen, ${ }^{1, *}$ Philippe Grelu, ${ }^{2, \dagger}$ and J. M. Soto-Crespo ${ }^{3, \ddagger}$ \\ ${ }^{1}$ Department of Physics, Southeast University, Nanjing 211189, China \\ ${ }^{2}$ Laboratoire Interdisciplinaire Carnot de Bourgogne, UMR No. 6303 associée au CNRS, Université de Bourgogne, \\ 9 avenue A. Savary, BP 47870, Dijon Cedex 21078, France \\ ${ }^{3}$ Instituto de Óptica, Consejo Superior de Investigaciones Científicas, Serrano 121, Madrid 28006, Spain
}

(Received 9 July 2013; published 6 January 2014)

\begin{abstract}
Exact explicit rogue-wave solutions of intricate structures are presented for the long-wave-short-wave resonance equation. These vector parametric solutions feature coupled dark- and bright-field counterparts of the Peregrine soliton. Numerical simulations show the robustness of dark and bright rogue waves in spite of the onset of modulational instability. Dark fields originate from the complex interplay between anomalous dispersion and the nonlinearity driven by the coupled long wave. This unusual mechanism, not available in scalar nonlinear wave equation models, can provide a route to the experimental realization of dark rogue waves in, for instance, negative index media or with capillary-gravity waves.
\end{abstract}

DOI: 10.1103/PhysRevE.89.011201

PACS number(s): 46.40.-f, 47.20.Ky, 47.35.-i, 47.52.+j

Because of their dramatic and potentially devastating manifestations, oceanic rogue waves have been the focus of intense research for more than a decade [1,2]. The roguewave terminology itself refers to the adverse surprise that is experienced when a transient giant wave of extreme amplitude or steepness is suddenly formed in the vicinity of a cruising ship [3]. In addition to being deployed into the safer and controllable environment of water tanks [4,5], rogue-wave research is also spreading widely to other disciplines that share general features of nonlinearity and complexity. These new avenues for rogue-wave research include fluid dynamics [6-8], nonlinear optics and lasers [9-12], plasma physics [13], and Bose-Einstein condensation [14]. The possibility to accede to a general understanding of rogue-wave formation is still an open question [15]. Nonetheless, the debate stimulates the comparison of predictions and observations between distinct areas, such as hydrodynamics and nonlinear optics, in situations where analogous dynamics can be identified through a common equation model. So far, the nonlinear Schrödinger (NLS) equation has played such a pivotal role.

The Peregrine soliton, predicted 30 years ago [16], is the simplest rogue-wave solution associated with the NLS equation and it has recently been observed experimentally in a water-wave tank [4], a multicomponent plasma [13], and an optical fiber [10]. It is of fundamental significance because it is robust [17] and serves as the prototypical rogue-wave profile in various experimental fields. Indeed, the Peregrine soliton, as opposed to ordinary solitons that can be traced over long propagation distances, is localized in both transverse and propagation dimensions, thus reflecting the seemingly unpredictable appearance of a rogue wave [4,18]. Mathematically, the Peregrine soliton and related rogue-wave solutions of higher-order [5] are always expressed by rational functions.

\footnotetext{
*cshua@seu.edu.cn

${ }^{\dagger}$ philippe.grelu@u-bourgogne.fr

†joto@io.cfmac.csic.es
}

While rogue-wave investigation is flourishing in several fields of science, there is a necessity to go beyond the standard NLS description in order to model important classes of physical systems in a relevant way. One recent development consists in including dissipative terms since a substantial supply of energy - from the wind in oceanography to the pump for laser cavities [19] - is generally required to drive roguewave formations $[12,20]$. Considering the extreme amplitude or steepness of a rogue wave, it also seems legitimate to include higher-order terms such as in the Hirota and the Sasa-Satsuma equations [21,22].

Another development of major importance consists in the study of coupled-wave systems, as numerous physical systems comprise interacting wave components of different modes, frequencies, or polarizations. When compared to scalar dynamical systems, vector systems generally allow energy transfer between their additional degrees of freedom, which potentially yields families of intricate vector rogue-wave solutions. Indeed, rogue-wave families with complicated rational forms have been recently found in the Davey-Stewartson equation [23], the coupled Manakov system [24], and the coupled Hirota equations [25]. Let us recall that the scalar NLS equation does not admit single dark-rogue-wave solutions, even in the case of a defocusing nonlinearity. Thus a major motivation in the analysis of nonlinear coupled systems is to find new rational solutions with a dark-rogue-wave counterpart that are relevant to practical physical systems.

Among coupled-wave dynamics, the long-wave-shortwave (LWSW) resonance is a general parametric process that manifests when the group velocity of the short (highfrequency) wave matches the phase velocity of the long (lowfrequency) wave. The LWSW resonance has been predicted in plasma physics [26] and nonlinear optics [27,28]. For example, in negative index media, it can be achieved through a three-wave-mixing process where the two degenerate optical waves (i.e., short waves) propagate in the negative index branch while the generated difference-frequency wave (i.e., long wave) resides in the positive index branch [28]. In hydrodynamics, the LWSW resonance can result from the interaction between capillary and gravity waves [29-31]. 
The fact that capillary rogue waves [6] were discovered in a recent experiment stimulates further the investigation of roguewave solutions for media manifesting LWSW resonance. This is the aim of the present work.

We report here the analytical derivation of a family of exact rogue-wave solutions of the LWSW generic model in an explicit and concise form. The evolution of the vector solutions along with the control parameter highlights original dark and bright rogue waves, which do not have any counterpart in scalar models such as the NLS equation. Finally, the robustness of the solutions herein is confirmed numerically.

The nonlinear interaction of the short wave (SW) $A$ and the long wave (LW) $U$ is modeled by the following two coupled equations, expressed in a normalized form [28]:

$$
\begin{aligned}
i \frac{\partial A}{\partial \xi}+\frac{1}{2} \frac{\partial^{2} A}{\partial \tau^{2}}+U A & =0, \\
\frac{\partial U}{\partial \xi}-\frac{\partial|A|^{2}}{\partial \tau} & =0,
\end{aligned}
$$

where $\xi$ and $\tau$ are the propagation distance and the retarded time, respectively. We have assumed that the complex envelope $A(\xi, \tau)$ and the LW field $U(\xi, \tau)$ (which is a real field in this model) are invariant in transverse space coordinates and we have neglected the term resulting from the cascaded $\chi^{(2)}$ nonlinearity [28]. For physical discussion, the first of Eqs. (1) is arranged into a form similar to the standard NLS equation, making it clear that its nonlinearity is driven by the LW field $U$ rather than by the Kerr term $|A|^{2}$.

We follow the standard Darboux dressing procedure as in Ref. [32] and obtain after some manipulations the fundamental rogue-wave solutions of Eq. (1) as

$$
\begin{gathered}
A(\xi, \tau)=A_{0}\left[1-\frac{i \xi+\frac{i \tau}{2 m-\omega}+\frac{1}{2(2 m-\omega)(m-\omega)}}{(\tau-m \xi)^{2}+n^{2} \xi^{2}+1 / 4 n^{2}}\right], \\
U(\xi, \tau)=b+2 \frac{n^{2} \xi^{2}-(\tau-m \xi)^{2}+1 / 4 n^{2}}{\left[(\tau-m \xi)^{2}+n^{2} \xi^{2}+1 / 4 n^{2}\right]^{2}},
\end{gathered}
$$

where the initial plane wave $A_{0}(\xi, \tau)$ is defined by its amplitude $a>0$, frequency $\omega$, and wave number $k$ :

$$
A_{0}(\xi, \tau)=a \exp (-i k \xi+i \omega \tau)
$$

with $k=\omega^{2} / 2-b$, and $b \geq 0$ is an arbitrary constant defining the background of the coupled LW field. The parameters $m$ and $n$ are real, defined by

$$
\begin{gathered}
m=\frac{1}{6}\left[5 \omega-\sqrt{3\left(\omega^{2}+\ell+\sigma / \ell\right)}\right], \\
n= \pm \sqrt{(3 m-\omega)(m-\omega)},
\end{gathered}
$$

with $\sigma=\frac{1}{9} \omega^{4}+6 \omega a^{2}, \rho=\frac{1}{2} \omega^{6}-\frac{1}{54}\left(27 a^{2}+5 \omega^{3}\right)^{2}$, and

$$
\ell= \begin{cases}-\left(\rho-\sqrt{\rho^{2}-\sigma^{3}}\right)^{1 / 3}, & \omega \leq-3 \omega_{n} \\ \left(-\rho+\sqrt{\rho^{2}-\sigma^{3}}\right)^{1 / 3}, & -3 \omega_{n}<\omega \leq \frac{3}{2} \omega_{n} .\end{cases}
$$

Here $\omega_{n} \equiv\left(2 a^{2}\right)^{1 / 3}$ and the parameter $\ell$ has been given in piecewise form to avoid any ambiguity. Obviously, the allowed regime of $\omega$ is $\omega \leq 3 \omega_{n} / 2$; otherwise $\ell$ and $n$ would be complex, thereby invalidating the solutions (2) and (3). We note from these rational solutions that the complex SW field envelope $A$ is characterized by a second-order polynomial of $\xi$ and $\tau$, whereas the real LW field $U$ involves a fourth-order polynomial. They involve intricate rogue-wave structures solely determined by $m$ and $n$ or, alternatively, by $a$ and $\omega$ (not by $b$ ).

Whereas the Peregrine soliton always has a central amplitude of $3 a$, the rogue waves (2) and (3) have variable $\omega$-dependent central amplitudes, given by

$$
|A(0,0)|=a\left|\frac{4 m-\omega}{2 m-\omega}\right|, \quad U(0,0)=b+8 n^{2} .
$$

As $\omega \rightarrow-\infty$, the central amplitude of the SW field $A$ reaches a maximum value of $3 a$, but falls to zero as $\omega=4 m$, i.e., $\omega=$ $\left(8 a^{2} / 3\right)^{1 / 3} \equiv \omega_{0}$. Correspondingly, we have $\left.U(0,0)\right|_{\omega \rightarrow-\infty}=$ $b$ and $\left.U(0,0)\right|_{\omega=\omega_{0}}=b+2\left(3 a^{4}\right)^{1 / 3}$ for the LW field. The maximum value of $U(0,0)$ occurs at $\omega=0$ and is found to be $\left.U(0,0)\right|_{\omega=0}=b+6 a^{4 / 3}$. As $\omega$ increases up to $3 \omega_{n} / 2$, both rogue waves fade away into respective background reservoirs and thus their central amplitudes equal $a$ and $b$, respectively.

The evolution of $|A(0,0)|$ and $U(0,0)$ along with these specific evolution characteristics has been depicted in Fig. 1. In this figure we also highlight typical complex rogue-wave profiles of the $\mathrm{SW}$ field $A$. Interestingly, as illustrated by the top two insets in Fig. 1, for $\omega<\omega_{0}$, the $S W$ rogue wave possesses two characteristic points $\left(\xi_{0}, \tau_{0}\right)$

$$
\xi_{0}= \pm \frac{\sqrt{2(4 m-\omega)(m-\omega)}}{4 n^{2}(2 m-\omega)}, \quad \tau_{0}=-(2 m-\omega) \xi_{0}
$$

which fulfill $\left|A\left(\xi_{0}, \tau_{0}\right)\right|=0$; we thereby term them the zeroamplitude points [25]. They are also $\omega$ dependent and thus different from the zeros of the Peregrine soliton, which are invariably given by $\left(0, \pm \frac{\sqrt{3}}{2 a}\right)$ (here $a$ is the background height) $[4,16]$. It is clear from Eq. (9) that as $\omega=\omega_{0}$, the zero-amplitude points merge into a single point $(0,0)$, but

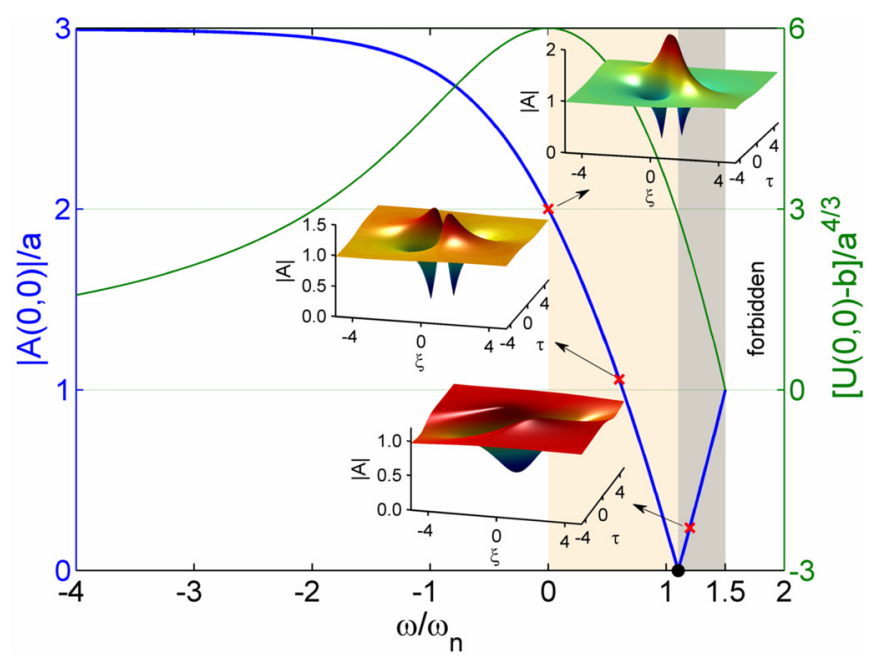

FIG. 1. (Color online) Evolution of $|A(0,0)|$ [dark gray (blue) line normalized to $a]$ and $[U(0,0)-b]$ [light gray (green) line normalized to $a^{4 / 3}$ ] with $\omega$ (normalized to $\omega_{n}$ ). The insets (from top to bottom) display the rogue-wave states of the SW field for $\omega=0$, $0.6 \omega_{n}$, and $1.2 \omega_{n}$, respectively, all with $a=1$. The rogue waves are forbidden in the region of $\omega>3 \omega_{n} / 2$. 


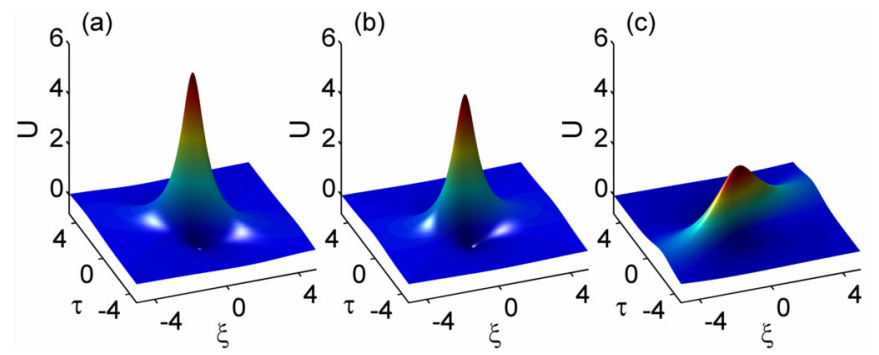

FIG. 2. (Color online) Rogue-wave states of the real LW field $U$ for specific $\omega$ values: (a) 0 , (b) $0.6 \omega_{n}$, and (c) $1.2 \omega_{n}$, all with $a=1$ and the same background $b=0$.

as $\omega_{0}<\omega<3 \omega_{n} / 2$, no zero-amplitude point will exist. As illustrated by the lower inset in Fig. 1, the rogue wave without zero-amplitude point features a single central hole and hence can be termed a dark rogue wave. Hence the SW rogue wave can be classified into bright, intermediate, and dark states corresponding to the domains $\omega \leq 0,0<\omega<\omega_{0}$, and $\omega_{0} \leq \omega<3 \omega_{n} / 2$, respectively. We can dub the rogue wave with a degenerate zero-amplitude point as a black rogue wave, in reminiscence of the black soliton terminology [33].

The LW rogue-wave component (3) features a minimum value of $U_{\min }=b-n^{2}$ occurring at $\left(0, \pm \frac{\sqrt{3}}{2|n|}\right)$. Hence, if the background height of the LW field, which does not affect the structure of the SW rogue wave, is chosen such as $b \geq 3 a^{4 / 3} / 4$, the nonlinearity induced by $U$ in Eq. (1) will be totally positive for all allowed $\omega$. Figure 2 displays the rogue-wave states of the LW field, each one corresponding to those shown in the insets in Fig. 1. It can be clearly seen that the rogue-wave structure, which is also independent of the value of $b$, always features a central hump that decreases in amplitude as $\omega$ grows.

Let us now discuss the specific physical mechanisms at play. We recall that in the scalar NLS equation, a self-defocusing Kerr nonlinearity results in a rational solution becoming singular, thus precluding a dark-rogue-wave solution. Here, owing to the complex interplay between anomalous dispersion and the nonlinearity resulting from the coupling between $A$ and $U$ fields, we anticipate that the contribution part of $U$ integrated along with propagation would wipe out the singularities, giving rise to an unusual dark-rogue-wave solution. In an intermediate rogue-wave regime (pink region in Fig. 1), the two characteristic zero-amplitude points can eventually merge owing to this special type of nonlinearity. Figure 3 illustrates the involved process through which a black-rogue-wave solution forms, when the parameter $\omega$ is varied. Specifically, as $\omega$ increases from 0 to $\omega_{0}$, the state of the SW rogue wave manifests as bright [Fig. 3(a)], intermediate [Figs. 3(b) and 3(c)], and black [Fig. 3(d)] structures, each characterized by the relative position of the two zero-amplitude points.

Field coupling provides a drastic change of the effective nonlinearity characterized by $U$. It also favors strongly asymmetric solutions. If we consider a symmetric bright pulse formed at a given time, it has both temporally increasing and decreasing intensity parts, initially symmetric. One can infer from Eq. (1) that, due to the coupling with $U$, the increasing intensity part of the pulse will induce an increase of $U$ with propagation, as in a self-focusing process, whereas the

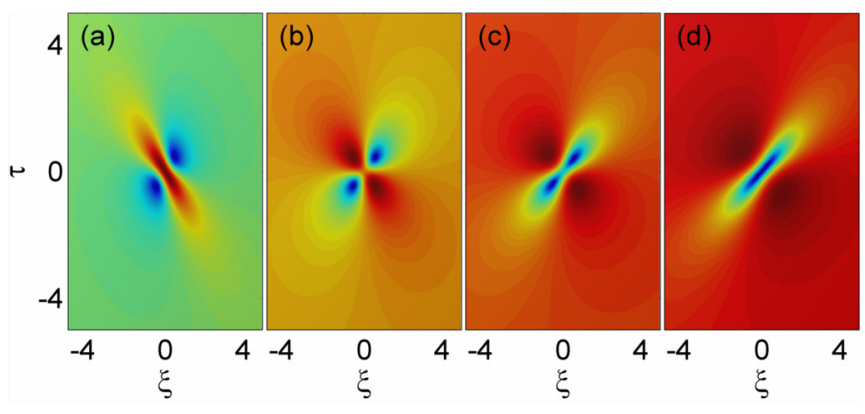

FIG. 3. (Color online) Contour plots for intermediate roguewave states with specific $\omega$ values: (a) 0 , (b) $0.6 \omega_{n}$, (c) $0.9 \omega_{n}$, and (d) $\omega_{0}$, all obtained under $a=1$ and $b=0$.

decreasing intensity part of the pulse will make a decrease of $U$ as in a self-defocusing process. This asymmetrizes the pulse in a peculiar way. Eventually, the temporal asymmetrization will give rise to a combination of bright and dark localized pulsed solutions, depending on the magnitude of the normalized frequency $\omega / \omega_{n}$.

We have also performed extensive numerical simulations to test whether the shapes of the analytical solutions (2) and (3) are preserved in the presence of a small amount of random noise. Specifically, we solved Eq. (1) numerically for initial pulse inputs given by Eqs. (2) and (3) at $\xi=-5$. We first checked that simulations reproduced exactly the ulterior behavior of the above analytical solutions. Then we perturbed the initial conditions by multiplying the real and imaginary parts of $A$ and $U$ by $\left[1+\epsilon f_{n}(\tau)\right](n=1,2,3)$, respectively. Here $f_{n}$ are three uncorrelated random functions uniformly distributed in the interval $[-0.5,0.5]$ and $\epsilon$ is a small number characterizing the strength of perturbations. The numerical results intended for the black rogue wave are illustrated in Fig. 4, where we
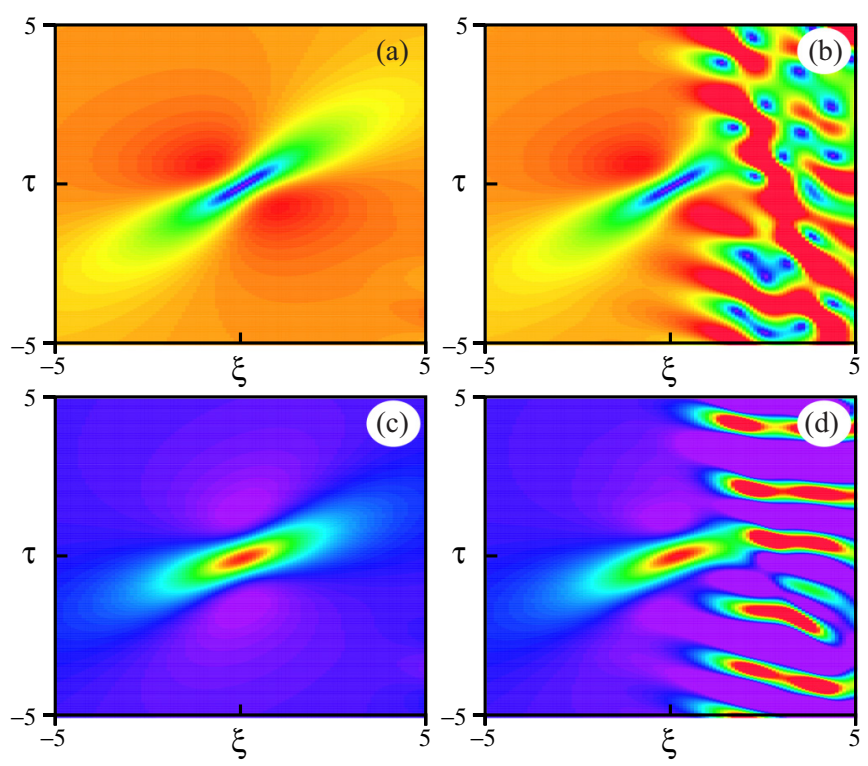

FIG. 4. (Color online) Numerical results intended for the black rogue wave $|A|$ and the coupled LW field $U$ by choosing $a=1$, $b=0$, and $\omega=\omega_{0} \approx 1.1 \omega_{n}$ : (a) and (c) unperturbed rogue waves $(\epsilon=0)$ and (b) and (d) perturbed situation with the noise strength $\epsilon=0.002$. 


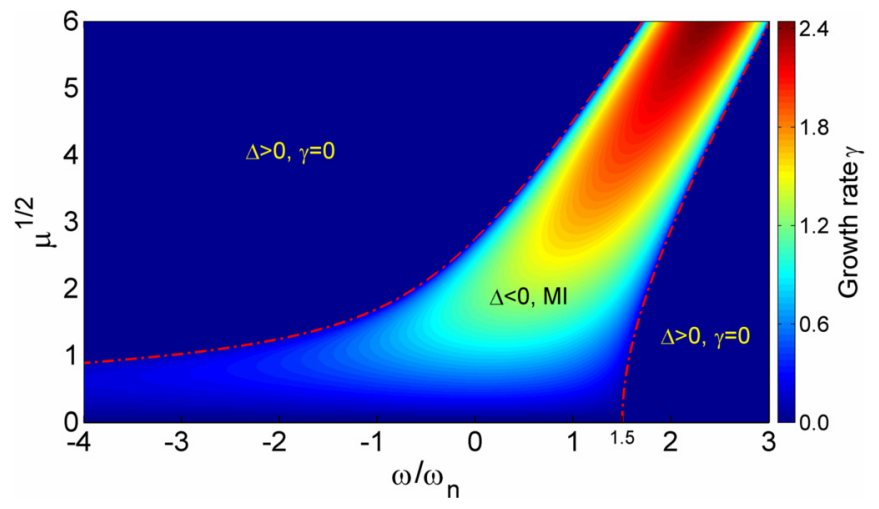

FIG. 5. (Color online) Linear stability diagram for the planewave solutions $A=A_{0}$ and $U=b$ with $a=1$. The red dash-dotted curves stand for the marginal stability defined by $\Delta=0$. The contour plot shows the growth rate $\gamma$ of the MI.

use $a=1, b=0, \omega=\omega_{0}$, and $\epsilon=0$ and 0.002. Figures 4(a) and $4(\mathrm{c})$ correspond to the unperturbed numerical solutions (i.e., $\epsilon=0$ ), clearly showing a very precise coincidence with our analytical solutions. When $\epsilon=0.002$ [see Figs. 4(b) and 4(d)], the numerical solutions begin as before, but the modulational instability (MI) of the background grows exponentially until it starts to interfere strongly with the localized solutions.

In fact, the MI of the background can be readily calculated $[31,32]$. We add small-amplitude Fourier modes to the plane-wave solutions and express them as $A=A_{0}\{1+$ $\left.p \exp [i \sqrt{\mu}(\tau-\kappa \xi)]+q^{*} \exp \left[-i \sqrt{\mu}\left(\tau-\kappa^{*} \xi\right)\right]\right\}$ and $U=$ $b+\delta \exp [i \sqrt{\mu}(\tau-\kappa \xi)]+\delta^{*} \exp \left[-i \sqrt{\mu}\left(\tau-\kappa^{*} \xi\right)\right]$, where $p, q$, and $\delta$ are small amplitudes of the Fourier modes. The propagation parameters $\mu$ and $\kappa$ are assumed to be positive and complex, respectively. A substitution of these perturbed solutions into Eq. (1) followed by linearization yields the dispersion relation

$$
\kappa(\kappa-\omega)^{2}-\mu \kappa / 4-a^{2}=0 .
$$

We know that the MI arises from a nonreal value of $\kappa$ and can be defined by the growth rate $\gamma=\sqrt{\mu} \operatorname{Im}(\kappa)>0$. Thus, to initiate it, the discriminant of Eq. (10),

$$
\Delta=\frac{1}{16} \mu^{3}-\frac{1}{2} \omega^{2} \mu^{2}-\omega\left(9 a^{2}-\omega^{3}\right) \mu-a^{2}\left(27 a^{2}-4 \omega^{3}\right),
$$

should be negative, i.e., $\Delta<0$. The marginal stability curves can be calculated from $\Delta=0$ for given $a$. Figure 5 shows the regime of MI for $a=1$, which is surrounded by two red dash-dotted curves and the line $\mu=0$. We also calculate the growth rate $\gamma$ in the whole $\left(\omega / \omega_{n}, \mu^{1 / 2}\right)$ plane, showing that the MI occurs within the $\Delta<0$ region. It can be clearly seen that within the existence region of our rogue waves, the growth rate $\gamma$ responsible for the MI can be very small for $\mu<1$. However, due to its exponential growth, MI can still develop to a large value after some distance and eventually interfere with the spatiotemporally localized rogue waves, as in Figs. 4(b) and 4(d). In addition, our analytical results for the MI exhibit consistency with the numerical simulations; from Fig. 4(d) the modulation period can be inferred to be around 10/5.5, corresponding to a modulation frequency of $\sqrt{\mu} \approx 3.5$, and the latter is almost the same frequency that the maximum $\gamma$ value at $\omega=\omega_{0}$ would correspond to, as seen in Fig. 5.

In conclusion, we have presented the exact explicit roguewave solutions of the LWSW equation governing the resonant interaction between long and short waves. We have checked numerically that the shapes of these rogue-wave solutions are preserved in the presence of a small amount of random noise. We showed that a dark rogue wave-the dark counterpart of the Peregrine soliton-can be formed as a result of an interplay between the anomalous dispersion and a positive nonlinearity due to the two-wave coupling, in the special case where two zero-amplitude points merge. This unusual mechanism, not available in the most-studied scalar NLS-type equation models $[4,16,21,22]$, can provide a possible route to the experimental realization of dark rogue waves in, for instance, negative index media [28] or with capillary-gravity waves [6,7,29-31]. In view of the universality of the LWSW resonance in many areas, with a particular emphasis on fluid dynamics and nonlinear optics, we anticipate that our work will open new research opportunities and may impact significantly on experimental design.

Note added. Recently, a mathematical derivation of similar analytical rogue-wave solutions of the LWSW equation was also obtained by Chow et al. using the Hirota bilinear method [34].

S.C. acknowledges support from the Qing Lan Project, the National Natural Science Foundation of China (Grant No. 11174050), and the Natural Science Foundation of Jiangsu Province of China (Grant No. BK2011586). Ph.G. was supported by the Agence Nationale de la Recherche (Projects No. ANR-2010-BLANC-0417-01 and No. ANR12-BS04-0011). The work of J.M.S.-C. was supported by MINECO under Contracts No. FIS2009-09895 and No. TEC2012-37958-C02-02.
[1] E. Pelinovsky and C. Kharif, Extreme Ocean Waves (Springer, Berlin, 2008).

[2] M. Onorato, S. Residori, U. Bortolozzo, A. Montina, and F. T. Arecchi, Phys. Rep. 528, 47 (2013).

[3] M. Onorato, D. Proment, G. Clauss, and M. Klein, PLoS ONE 8, e54629 (2013).

[4] A. Chabchoub, N. P. Hoffmann, and N. Akhmediev, Phys. Rev. Lett. 106, 204502 (2011).
[5] A. Chabchoub, N. Hoffmann, M. Onorato, and N. Akhmediev, Phys. Rev. X 2, 011015 (2012).

[6] M. Shats, H. Punzmann, and H. Xia, Phys. Rev. Lett. 104, 104503 (2010).

[7] M. Shats, H. Xia, and H. Punzmann, Phys. Rev. Lett. 108, 034502 (2012).

[8] H. Xia, T. Maimbourg, H. Punzmann, and M. Shats, Phys. Rev. Lett. 109, 114502 (2012). 
[9] D. R. Solli, C. Ropers, P. Koonath, and B. Jalali, Nature (London) 450, 1054 (2007).

[10] B. Kibler, J. Fatome, C. Finot, G. Millot, F. Dias, G. Genty, N. Akhmediev, and J. M. Dudley, Nat. Phys. 6, 790 (2010).

[11] R. Driben and I. Babushkin, Opt. Lett. 37, 5157 (2012).

[12] C. Lecaplain, Ph. Grelu, J. M. Soto-Crespo, and N. Akhmediev, Phys. Rev. Lett. 108, 233901 (2012).

[13] H. Bailung, S. K. Sharma, and Y. Nakamura, Phys. Rev. Lett. 107, 255005 (2011).

[14] Yu. V. Bludov, V. V. Konotop, and N. Akhmediev, Phys. Rev. A 80, 033610 (2009).

[15] A. Demircan, S. Amiranashvili, C. Brée, C. Mahnke, F. Mitschke, and G. Steinmeyer, Sci. Rep. 2, 850 (2012).

[16] D. H. Peregrine, J. Aust. Math. Soc. Ser. B 25, 16 (1983).

[17] A. Ankiewicz, N. Devine, and N. Akhmediev, Phys. Lett. A 373, 3997 (2009).

[18] N. Akhmediev, A. Ankiewicz, and M. Taki, Phys. Lett. A 373, 675 (2009).

[19] Ph. Grelu and N. Akhmediev, Nat. Photon. 6, 84 (2012).

[20] J. M. Soto-Crespo, Ph. Grelu, and N. Akhmediev, Phys. Rev. E 84, 016604 (2011).
[21] A. Ankiewicz, J. M. Soto-Crespo, and N. Akhmediev, Phys. Rev. E 81, 046602 (2010).

[22] U. Bandelow and N. Akhmediev, Phys. Rev. E 86, 026606 (2012).

[23] Y. Ohta and J. Yang, Phys. Rev. E 86, 036604 (2012).

[24] F. Baronio, A. Degasperis, M. Conforti, and S. Wabnitz, Phys. Rev. Lett. 109, 044102 (2012).

[25] S. Chen and L.-Y. Song, Phys. Rev. E 87, 032910 (2013).

[26] V. E. Zakharov, Zh. Eksp. Teor. Fiz. 62, 1745 (1972) [Sov. Phys. JETP 35, 908 (1972)].

[27] Yu. S. Kivshar, Opt. Lett. 17, 1322 (1992).

[28] A. Chowdhury and J. A. Tataronis, Phys. Rev. Lett. 100, 153905 (2008).

[29] D. J. Benney, Stud. Appl. Math. 56, 81 (1977).

[30] V. D. Djordjevic and L. G. Redekopp, J. Fluid Mech. 79, 703 (1977).

[31] Y.-C. Ma and L. G. Redekopp, Phys. Fluids 22, 1872 (1979).

[32] O. C. Wright, III, Stud. Appl. Math. 117, 71 (2006).

[33] Yu. S. Kivshar and B. Luther-Davies, Phys. Rep. 298, 81 (1998).

[34] K. W. Chow, H. N. Chan, D. J. Kedziora, and R. H. J. Grimshaw, J. Phys. Soc. Jpn. 82, 074001 (2013). 